Check for updates

Cite this: Chem. Sci., 2018, 9, 6844

¿ All publication charges for this article have been paid for by the Royal Society of Chemistry

\title{
Glass wool: a novel support for heterogeneous catalysis $\dagger$
}

\author{
Ayda Elhage, (D) Bowen Wang, (D) Nancy Marina, M. Luisa Marin, (D) $\$$ Menandro Cruz, \\ Anabel E. Lanterna (D) and Juan C. Scaiano (DD *
}

\begin{abstract}
Heterogeneous catalysis presents significant advantages over homogeneous catalysis such as ease of separation and reuse of the catalyst. Here we show that a very inexpensive, manageable and widely available material - glass wool - can act as a catalyst support for a number of different reactions. Different metal and metal oxide nanoparticles, based on $\mathrm{Pd}, \mathrm{Co}, \mathrm{Cu}, \mathrm{Au}$ and $\mathrm{Ru}$, were deposited on glass wool and used as heterogeneous catalysts for a variety of thermal and photochemical organic reactions including reductive de-halogenation of aryl halides, reduction of nitrobenzene, $\mathrm{Csp}^{3}-\mathrm{Csp}^{3}$ couplings, $\mathrm{N}-\mathrm{C}$ heterocycloadditions (click chemistry) and Csp-Csp ${ }^{2}$ couplings (Sonogashira couplings). The use of glass wool as a catalyst support for important organic reactions, particularly $\mathrm{C}-\mathrm{C}$ couplings, opens the opportunity to develop economical heterogeneous catalysts with excellent potential for flow photochemistry application.
\end{abstract}

Received 14th May 2018

Accepted 10th July 2018

DOI: $10.1039 / \mathrm{c} 8 \mathrm{sc} 02115 \mathrm{e}$

rsc.li/chemical-science

properties and easy to modify to provide physical or chemical affinity towards many catalytic materials. Glass wool is widely employed for thermal and noise insulation in homes, appliances and instrumentation. In chemistry, GW is commonly used as a filter, packing material in GC columns, purge trap and adsorbent bed, ${ }^{8}$ where it is normally regarded as a fairly robust inert material towards many chemicals, showing advantages including good $\mathrm{pH}$ tolerance. There are some examples where GW is used as an attachment surface for applications in biology, including bactericidal studies, ${ }^{\mathbf{9 , 1 0}}$ but its applications in organic chemistry are virtually unexplored. The use of glass fibers and cloths for catalysis was reviewed in $2002 ;^{11}$ their applications at the time dealt with gas and liquid processes, mostly oxidations, but interestingly it was recognized that these materials could avoid the technological problems and limitations associated with handling and separation of powders. While this contribution was in preparation, Barelko et al. ${ }^{12}$ published a review with the significant technological advances achieved during the last 15 years. Yet, with the exception of a nitro-compound reduction, no applications to organic chemistry were reported, in particular, not a single example on $\mathrm{C}-\mathrm{C}$ bond formation, a key reaction in organic synthesis and drug-development applications. Some concerns about the inert nature of the glass wool were noted many years ago by Hayes and Macdonald, but no further studies were found in the literature. ${ }^{13}$ Apart from these limited examples in catalysis, where high temperature ${ }^{14}$ or redox harsh conditions are usually employed, ${ }^{\mathbf{1 2}}$ it is hard to find GW uses other than the ones mentioned above, ${ }^{8}$ largely excluding organic catalysis. There are few studies focused on depositing metals on glass, mostly dedicated to redox reactions, ${ }^{15-17}$ however to the best of our knowledge, no complex 


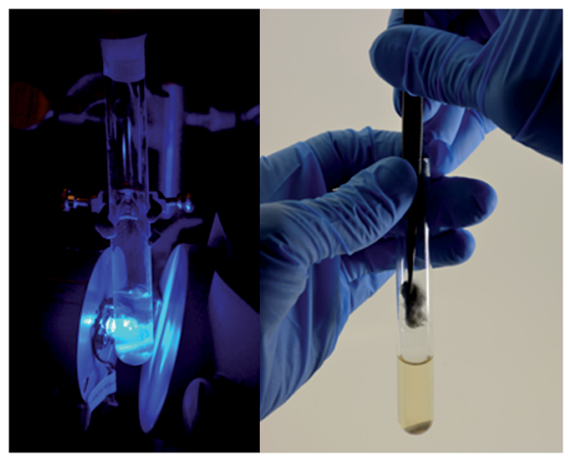

Fig. 1 Picture showing the reaction setup used under LED irradiation and continuous stirring (left), and the easy removal of the GW material from the reaction vessel utilizing a pair of tweezers (right).

organic reactions - such as $\mathrm{C}-\mathrm{C}$ couplings - have been explored utilizing glass as a catalyst support.

In this contribution, we report a number of metal and metal oxide nanostructures supported on commercially available sometimes modified - GW and how they perform on a variety of catalytic processes. We explored individual reactions emphasizing the use of classic reactions with diverse novel materials. Our work on glass wool was initiated with the assumption that this support would be of the passive type. Notice that the use of these materials extremely facilitates the catalyst separation from the reaction vessel, and thus a regular pair of tweezers can be used to remove the GW as shown in Fig. 1. Further purification by filtration is straightforward.

\section{Results and discussion}

\section{Materials characterization}

Two different types of commercial glass wool were used in this work: non-silanized (NGW) and silanized (SGW) glass wool. They were decorated with different metal and metal oxide nanoparticles, namely $\mathrm{Au}, \mathrm{Pd}, \mathrm{Ru}, \mathrm{Co}$ and $\mathrm{Cu}$, utilizing photoinduced and chemical methods as described in the ESI. $\dagger$ The optimal synthetic method was chosen based on the observed catalytic activity and stability of the new materials prepared. Thus, GWs were subjected to different pre-treatments before incorporating the metal/metal oxide nanoparticles in order to efficiently attach the metal to the GW surface. The experimental section summarizes several different trials and the rationale behind the selection of the preferred pre-treatment method. In particular, APTES treatment was selected to add a source of amino groups on the glass surface. These groups not only help to attach metal nanoparticles to the glass surface but can also be used as reducing agents for in situ formation of metal nanoparticles under dark conditions (see ESI $\dagger$ ). ${ }^{18}$ In general, non-silanized and silanized GW show no difference in their catalytic activity. In particular, NGW is preferred in cases were modification with APTES is required. When only acid pretreatment is used, SGW shows better reactivity (vide infra). Fig. 2 shows the materials before and after different surface modifications.
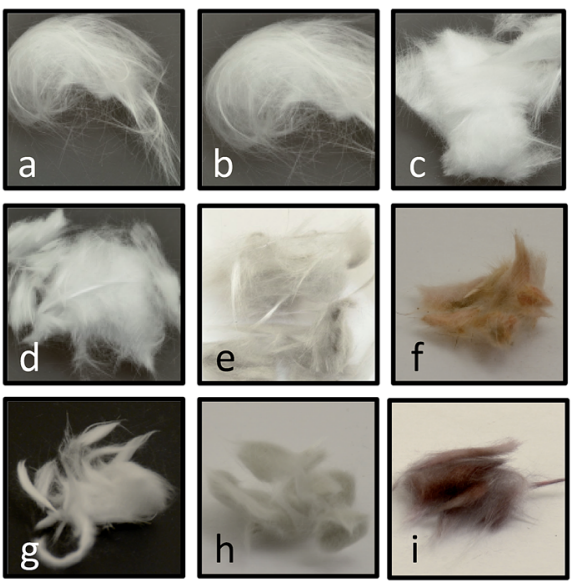

Fig. 2 Pictures of different materials used in this work: (a) pristine SGW, (b) pristine NGW, (c) HCl-treated SGW, d) APTES-functionalized NGW (NGW*), (e) Pd@SGW, (f) Cu@NGW, (g) Co@SGW, (h) Ru@NGW* and (i) Au@NGW*

The microscopic analysis of the GW samples was also performed. As shown in Fig. 3a, the commercial GW is constituted by glass fibers of about $10 \mu \mathrm{m}$ diameter. The crystalline structures present together with the fibers correspond to $\mathrm{NaCl}$ as determined by a careful EDS analysis of different areas of the image (Fig. $3 \mathrm{~b}$ and c). Upon derivatization, the presence of metal or metal oxides nanoparticles can be easily distinguished by SEM. Fig. 3d and e show the surface of a glass fiber covered by $\mathrm{Co}_{x} \mathrm{O}_{y}$ NPs and the corresponding EDS spectra. The same characterization was performed for each of the different metalderivatized GWs as shown in Fig. S5-S8.†

The materials were further characterized by ICP-OES, diffuse reflectance (DR) and X-ray photoelectron spectroscopy (XPS). The amount of metal loaded on each material as well as particle
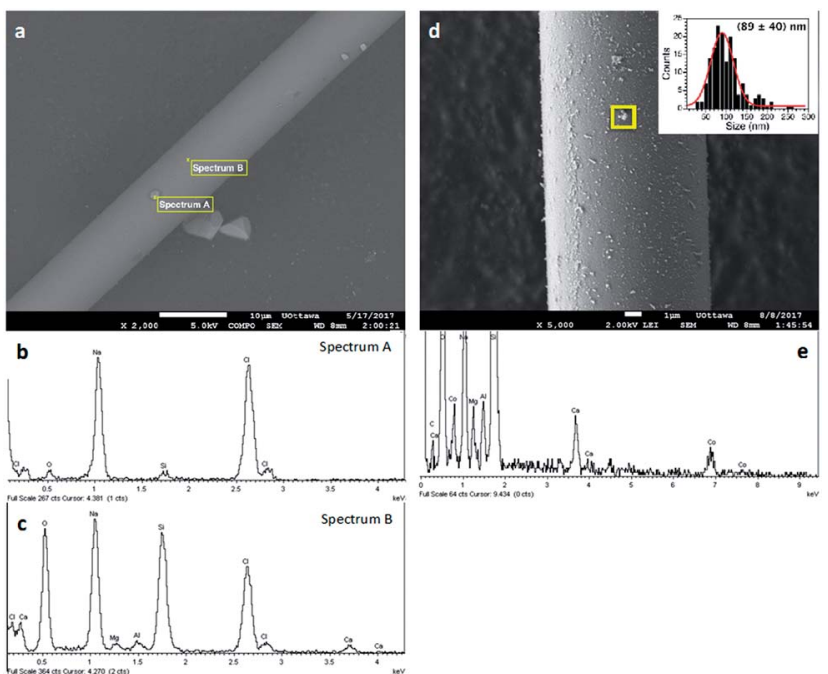

Fig. 3 SEM images (top) and EDS spectra (bottom) in the marked areas of untreated SGW $(a-c)$ and of Co@SGW (d and e). Notice that the particulates on the SGW fiber are mostly composed of $\mathrm{NaCl}$. Agglomerated particles were not considered to determine particle size distribution. 
size distribution and calculated surface area are reported in Table 1. Fig. 4 (left) shows the absorption profile of SGW before and after Co-derivatization, where slight changes on the absorption of Co@SGW can account for the contribution of $\mathrm{Co}_{x} \mathrm{O}_{y}$ species in the material. The different absorption profiles of other metal-derivatized GWs are presented in Fig. S9-S12, $\dagger$ where Pd, Au and Ru-derivatized GW show higher absorption in the visible region compared to the non-derivatized GW. XPS deconvolution analyses were performed for each material in order to determine the oxidation state of each metal. Fig. 4 (right) shows the deconvoluted Co 2p HR-XPS spectrum obtained for Co@SGW, whereas the rest of the materials are described in Fig. S13-S16. $\dagger$ Characteristic peaks for Co 2 p were fitted using the spin-orbit split constituted by Co $2 \mathrm{p}_{3 / 2}$ (781.2 $\mathrm{eV})$ and Co $2 \mathrm{p}_{1 / 2}(797.7 \mathrm{eV})$ separated by $\sim 16.5 \mathrm{eV}$. The corresponding Co(II) satellites (787.4 and $803.5 \mathrm{eV}$ ) further prove the presence of Co oxides $\left(\mathrm{Co}_{x} \mathrm{O}_{y}\right) \cdot{ }^{19}$ XPS analysis of the Pd@NGW* exposes a Pd 3d core-level spectrum deconvoluted by using two spin-orbit split Pd $3 \mathrm{~d}_{5 / 2}$ and $\mathrm{Pd} 3 \mathrm{~d}_{3 / 2}$ components centered at $337 \mathrm{eV}$ and $342.4 \mathrm{eV}$ and separated by $\sim 5.4 \mathrm{eV}$, which more likely correspond to PdO. ${ }^{4}$ In contrast, more reduced Pd species are found in the Pd@SGW material with spin-orbit components at $335 \mathrm{eV}$ and $340.4 \mathrm{eV}$. The XPS fitting for the $\mathrm{Ru} 3 \mathrm{p}$ in Ru@NGW reveals the presence of two main peaks at 463.1 and $485.5 \mathrm{eV}$, which according to the literature correspond to $\mathrm{RuO}_{2},{ }^{20}$ although other reports suggest a mixture between

Table 1 Characterization of the metal content on the metal-derivatized GWs

\begin{tabular}{lccl}
\hline Material $^{a}$ & $\begin{array}{l}\text { Metal amount } \\
(\mathrm{wt} \%)\end{array}$ & $\begin{array}{l}\text { Particle } \\
\operatorname{size}^{c}(\mathrm{~nm})\end{array}$ & $\begin{array}{l}\text { Surface } \\
\mathrm{area}^{d}\left(\mathrm{~cm}^{2} \mathrm{~g}^{-1}\right)\end{array}$ \\
\hline Pd@SGW & $0.16 \pm 0.03$ & $19 \pm 8$ & 425 \\
Pd@NGW & $0.54 \pm 0.05$ & $22 \pm 9$ & 1184 \\
Co@SGW & $0.070 \pm 0.005$ & $89 \pm 40$ & 53 \\
Ru@NGW* & $0.030 \pm 0.001$ & $63 \pm 30$ & 23 \\
Cu@NGW* & $1.1 \pm 0.2$ & $100 \pm 60$ & 737 \\
Au@NGW* & $0.9 \pm 0.2$ & $23 \pm 10$ & 1215
\end{tabular}

${ }^{a}$ APTES treated GW are denoted with $*{ }^{b}$ By ICP-OES analysis. None of the metals mentioned were detected on $\mathrm{NGW}^{*}$, pristine NGW or pristine SGW. ${ }^{c}$ Particle size distributions were calculated without considering agglomerated particles. ${ }^{d}$ Metal surface per gram of glass wool; considering that the mean diameter of the GW fibers used is $10 \mu \mathrm{m}$, the GW $\left(d=2.2 \mathrm{~g} \mathrm{~cm}^{-3}\right)$ surface area is approximated to $1820 \mathrm{~cm}^{2} \mathrm{~g}^{-1}$.
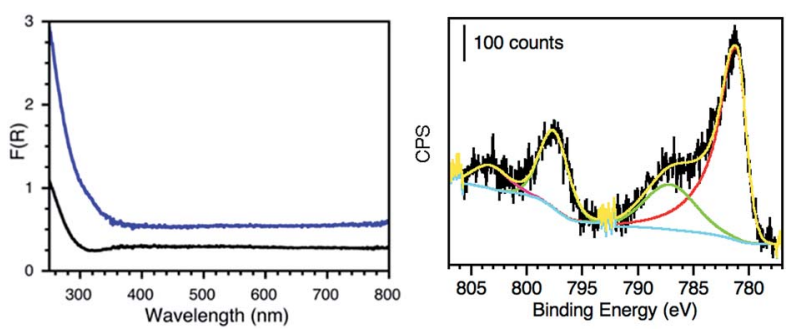

Fig. 4 Left: diffuse reflectance spectrum of HCl-treated SGW (black) and Co@SGW (blue). Right: deconvoluted Co $2 p$ HR-XPS spectrum of the Co@SGW catalyst. metallic $\mathrm{Ru}(0), \mathrm{RuO}_{2}$ and $\mathrm{RuO}_{2} \cdot x \mathrm{H}_{2} \mathrm{O}^{21,22}$ In the case of $\mathrm{Cu} @ N G W$, the two spin-orbit split $\mathrm{Cu} 2 \mathrm{p}_{3 / 2}(934.0 \mathrm{eV})$ and $\mathrm{Cu}$ $2 \mathrm{p}_{1 / 2}(953.7 \mathrm{eV})$ components $(\Delta \sim 19.7 \mathrm{eV})$ and the noticeable $\mathrm{Cu}(\mathrm{II})$ satellites indicate that $\mathrm{CuO}^{23}$ is present in this material. Finally, for $\mathrm{Au} @ \mathrm{NGW}^{*}$ the peaks corresponding to $\mathrm{Au} 4 \mathrm{f}_{7 / 2}(84.5$ $\mathrm{eV})$ and $\mathrm{Au} 4 \mathrm{f}_{5 / 2}(88.0 \mathrm{eV})$ suggest the presence of $\mathrm{Au}(0)$. The additional features at lower BE $(\sim 82 \mathrm{eV})$ can account for lowcoordinated atoms on the $\mathrm{Au}$ surface. ${ }^{24}$

\section{Catalytic activity}

In order to determine if these new materials would perform as catalysts we selected different reactions based on some of our previous reports. ${ }^{4,6,7,25}$ Scheme 1 summarizes the reactions tested that were successfully catalyzed utilizing the various new materials.

Conditions explored for each reaction vary from thermal control to photo-induced catalysis. Table 2 shows a summary of the reactivity of different GW-based materials toward different organic transformation, where the last column shows the table number and detailed entry for relevant examples. To discuss their activity we divided the following sections according to the organic transformation under study.

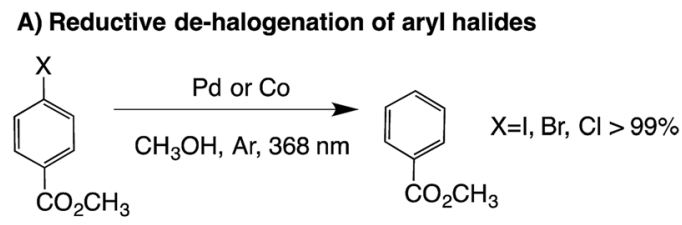

B) Reduction of nitrobenzene: to aniline

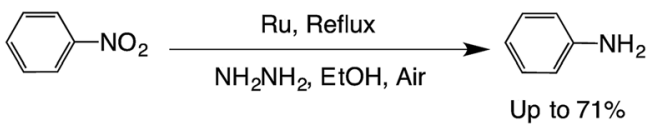

B') Reduction of nitrobenzene: to azo compounds

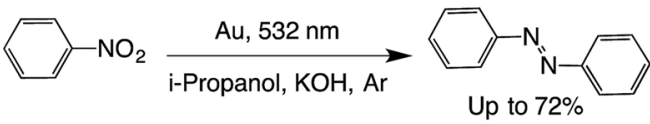

C) C-C coupling $\left(\mathrm{sp}^{3}-s \mathrm{p}^{3}\right)$ : dimerization of benzyl bromides

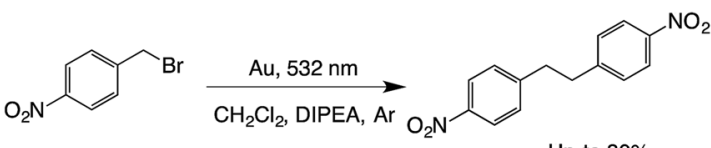

Up to $80 \%$

D) N-C heterocycloaddition: copper(I)-catalyzed azide alkyne cycloaddition

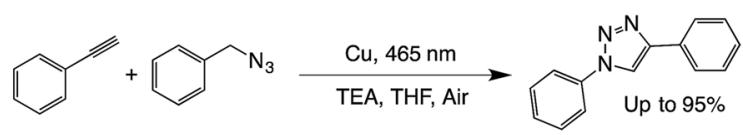

E) C-C coupling (sp-sp²): Sonogashira coupling

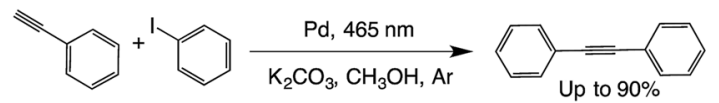

Scheme 1 Scope of reactions tested with the modified glass wool composites. Only the reactions that were successfully catalyzed are listed here. 
Table 2 Summary of the best reactivities observed when various GW-based materials are used as catalysts for different organic transformations

\begin{tabular}{|c|c|c|c|c|}
\hline Entry & Material $^{a}$ & Reaction & $\%$ Yield & Table (entry) \\
\hline $\mathrm{i}$ & Co@SGW & (A) Reductive de-halogenation & $>99$ & $3(\mathrm{i}, \mathrm{v})$ \\
\hline ii & Pd@SGW & & $>99$ & 3 (ii, vi) \\
\hline iv & $\mathrm{Au} @ \mathrm{NGW}^{*}$ & $\left(\mathrm{~B}^{\prime}\right)$ Reduction of nitrobenzene to azobenzene & 72 & 5 (ii) \\
\hline $\mathrm{v}$ & $\mathrm{Au} @ \mathrm{NGW}^{*}$ & (C) $\mathrm{C}-\mathrm{C}$ coupling $\left(\mathrm{sp}^{3}-\mathrm{sp}^{3}\right)$ & 80 & 6 (ii) \\
\hline vi & Cu@NGW $\left(\mathrm{NGW}^{*}\right)$ & (D) $\mathrm{N}-\mathrm{C}$ heterocycloaddition & 92 & 7 (i) \\
\hline
\end{tabular}

${ }^{a}$ The star $(*)$ indicates APTES-treated materials.

\section{Reductive de-halogenation of aryl halides}

De-halogenation processes are usually dictated by the Chalogen bond strength; thus, $\mathrm{C}-\mathrm{Cl}$ bonds are harder to break than $\mathrm{C}-\mathrm{Br}$ bonds and $\mathrm{C}-\mathrm{I}$ bonds, which is usually reflected under harsher reaction conditions to achieve de-halogenation. ${ }^{\mathbf{2 6 , 2 7}}$ Recent reports on dehalogenation processes involved the use of either high pressure conditions $\left(\mathrm{H}_{2}, 30 \mathrm{bar}\right),{ }^{28}$ or toxic and expensive iridium complexes. ${ }^{29}$ Here we performed photodehalogenation of methyl 4-halogenobenzoates catalyzed by GW-based materials, particularly, Pd@SGW, Co@SGW and SGW. Both Pd- and Co-based NPs are known to work as photocatalysts under UV-visible light..$^{30-33}$

The results summarized in Table 3 show that the reaction in the presence of Co@SGW proceeds under irradiation and can be accelerated under an inert atmosphere. Pd@SGW and SGW show the same reactivity as Co@SGW when methyl 4-iodobenzoate is used. As expected, the reaction slows down moving from iodide to bromide and to chloride reagents. This tendency is more evident with catalysts such as Pd@SGW and SGW, whereas Co@SGW has shown exceptional yields, being able to

Table 3 Light induced reductive dehalogenation catalysed by Co- and $\mathrm{Pd}$-derivatized $\mathrm{SGW}^{a}$

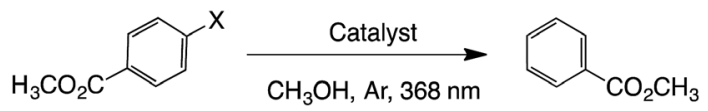

\begin{tabular}{lllll}
\hline & $\mathrm{X}^{b}(1)$ & Catalyst & Time (h) & $\%$ Yield $^{c}$ \\
\hline i & Br- & Co@SGW & 2 & $>99$ \\
ii & Br- & Pd@SGW & 2 & $>99$ \\
iii & Br- & SGW & 5 & $>99$ \\
ix & Br- & None & 5 & 98 \\
v & Cl- & Co@SGW & 3 & $>99$ \\
vi & Cl- & Pd@SGW & 20 & $>99$ \\
vii & Cl- & SGW & 24 & 37 \\
viii & Cl- & None & 24 & 36
\end{tabular}

${ }^{a}$ Reaction conditions: $0.2 \mathrm{mmol}$ of substrate $1,0.36 \mathrm{mmol}$ of $\mathrm{K}_{2} \mathrm{CO}_{3}$, $5 \mathrm{~mL}$ methanol, and $60 \mathrm{mg}$ catalyst under Ar. ${ }^{b}$ When $\mathrm{X}=\mathrm{I}$, yield of $99 \%$ is reached in the presence of Co@SGW (or SGW) in $15 \mathrm{~min}$ of irradiation under $\mathrm{Ar}$ or $1 \mathrm{~h}$ under air. Only $15 \%$ yield was detected after $24 \mathrm{~h}$ of reaction in the dark at $47{ }^{\circ} \mathrm{C}$. ${ }^{c}$ Yields were determined by GC-FID using $t$-stilbene as the external standard. reduce methyl 4-chlorobenzoate within $3 \mathrm{~h}$ (entry v, see $\mathrm{ESI} \dagger$ for kinetic analysis). Additionally, Co@SGW showed great reusability; thus, after 3 catalytic cycles $>99 \%$ yield of reductive compound was achieved within $4 \mathrm{~h}$ of irradiation of methyl 4 chlorobenzoate (Table S1†).

\section{Reduction of nitro compounds}

The photoreduction of nitrocompounds has been studied as a method to synthesize aniline derivatives under mild conditions. ${ }^{34,35}$ Based on our previous work ${ }^{\mathbf{1 8}}$ we decided to test the ability of Ru- and Au-derivatized GW as catalysts for the reduction of nitrobenzene to produce aniline. We used the optimized conditions previously reported for this type of organic transformation ${ }^{\mathbf{1 8 , 3 6}}$ (see the Experimental section). Table 4 summarizes the results of the nitrobenzene reduction by Ru-derivatized GWs. We noticed that using $\mathrm{NGW}^{*}$ or SGW* as supports shows no difference in the catalytic performance of the $\mathrm{Ru}$ catalyst. It is worth mentioning that Au-derivatized GW showed no catalytic activity under the same experimental conditions. The reaction kinetics are depicted in Fig. S3. $\dagger$

The formation of azo compounds as products of this reaction is also possible. However, their formation under illumination is rarely found as the reductive environment forces the reaction

Table 4 Reduction of nitrobenzene to aniline catalysed by Ru-derivatized $\mathrm{GW}^{a}$

\begin{tabular}{|c|c|c|}
\hline Catalyst & Time (h) & $\%$ Yield $^{b}$ \\
\hline Ru@SGW* & 24 & 28 \\
\hline Ru@SGW* & 64 & $71^{c}$ \\
\hline Ru@NGW* & 24 & 28 \\
\hline Ru@NGW* & 64 & $66^{d}$ \\
\hline $\mathrm{NGW}^{*}$ & 24 & ND \\
\hline None & 24 & ND \\
\hline
\end{tabular}

${ }^{a}$ Reaction conditions: $25 \mathrm{mg}$ of catalyst, $1.5 \mathrm{mmol}$ nitrobenzene, 6 eq. hydrazine, and $5 \mathrm{~mL}$ ethanol. ${ }^{b}$ Yields were determined by GC-FID using 1,3,5-trimethoxybenzene as an internal standard. ${ }^{c}$ After 4 days $85 \%$ of the desired product was detected. ${ }^{d}$ After 4 days $79 \%$ of the desired product was detected. 
toward the corresponding amine. ${ }^{37}$ There are just a few examples where the azo-compounds form under illumination of AuNP. ${ }^{36,38,39}$ Here we show that excitation of AuNPs deposited on GW can also catalyze this reaction under green light irradiation for $24 \mathrm{~h}$ with moderate yields (Table 5). Briefly, the catalyst was mixed together with the nitrocompound in i-propanol under an inert atmosphere (Ar) in the presence of $\mathrm{KOH}$ and irradiated with $532 \mathrm{~nm}$ LEDs. The formation of the azocompound was monitored by UV-Vis spectroscopy (Fig. S4 $\dagger$ ) following the azobenzene absorption band at $\sim 350 \mathrm{~nm}$. As expected when visible light is utilized only trans azobenzene is detected as the reaction product. $^{40}$

Combining the data presented in Tables 3 and 4 it becomes clear that GW-based catalysts offer an excellent opportunity of product and selectivity control by tuning the metal, its loading and the type of activation used (i.e. thermal or photochemical activation).

\section{C-C coupling $\left(\mathrm{sp}^{3}-\mathrm{sp}^{3}\right)$}

The reductive dimerization of benzyl bromides can be photoinduced using supported AuNPs and green light excitation. ${ }^{6}$ This reaction is known as a radical reaction in which light intensity can lead the selectivity towards the dimer product, and thus, higher intensity yields more dimer. Much to our surprise the same reaction proceeds with excellent yields if AuNPs are deposited on GW (Table 6). It is worth highlighting that a noticeable product yield is obtained in the absence of catalyst under these irradiation conditions. These have been previously reported as a contribution from the UV contamination of the green light source. ${ }^{6}$ The material also shows reasonable reusability (see Table S1†).

\section{N-C heterocycloaddition (click reaction).}

We have recently reported the photocatalyzed click reaction using supported $\mathrm{Cu}_{x} \mathrm{O}$ nanoparticles on $\mathrm{TiO}_{2}$ and $\mathrm{Nb}_{2} \mathrm{O}_{5} \cdot{ }^{7}$ The reaction can proceed within $6 \mathrm{~h}$ under both UV and visible light. The unexpected reactivity of the material under visible light suggests that the $\mathrm{Cu}_{x} \mathrm{O}$ particles play an important light

Table 5 Light induced reduction of nitrobenzene to azobenzene catalyzed by Au@NGW*a

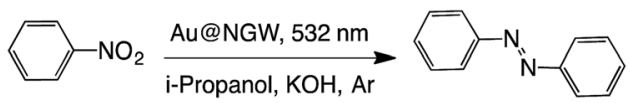

\begin{tabular}{lllll}
\hline & Catalyst & Time (h) & \% Conv & \% Yield $^{b}$ \\
\hline i & Au@NGW* & 24 & 68 & 65 \\
ii & Au@NGW* & 48 & 73 & 72 \\
iii & Au@NGW ${ }^{* c}$ & 24 & 40 & 35 \\
iv & Au@NGW ${ }^{*} d$ & 24 & ND & ND \\
v & NGW $^{*}$ & 24 & ND & ND \\
vi & None & 24 & ND & ND
\end{tabular}

${ }^{a}$ Reaction conditions: $0.3 \mathrm{mmol}$ of substrate, $0.03 \mathrm{mmol}$ of $\mathrm{KOH}, 5 \mathrm{~mL}$ ipropanol, and $60 \mathrm{mg}$ catalyst under Ar. ${ }^{b}$ Conversion and yields were determined by UV-Vis spectroscopy. ${ }^{c}$ In the dark at $85{ }^{\circ} \mathrm{C} .{ }^{d}$ Under air.
Table 6 Light induced reductive dimerization of p-nitrobenzyl bromide catalyzed by Au@NGW*a

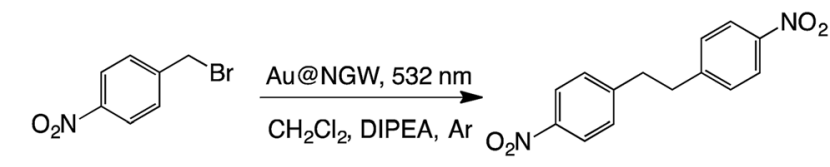

\begin{tabular}{lllll}
\hline & Catalyst & Time (h) & \% Conv & \% Yield $^{c}$ \\
\hline i & Au@NGW* & 5 & 77 & 64 \\
ii & Au@NGW* & 7 & 100 & 80 \\
iii & Au@NGW*d & 48 & ND & ND \\
iv & NGW $^{*}$ & 24 & 36 & 26 \\
v & None $^{e}$ & 5 & - & 25 \\
vi & None $^{e}$ & 5 & - & ND
\end{tabular}

${ }^{a}$ Reaction conditions: $0.1 \mathrm{mmol}$ of substrate, 2 eq. of DIPEA, and $30 \mathrm{mg}$ of catalyst in $4 \mathrm{~mL}$ of $\mathrm{CH}_{2} \mathrm{Cl}_{2} \cdot{ }^{b}$ By-product: 4-nitrotoluene. ${ }^{c}$ Yields and conversions were calculated by ${ }^{1} \mathrm{H}$-NMR using caffeine as the external standard. ${ }^{d}$ Dark reaction at $39{ }^{\circ} \mathrm{C} .{ }^{e}$ From ref. 6 .

harvesting role. With this in mind, we decided to test the $\mathrm{Cu}_{x} \mathrm{O}$ activity when deposited on GW. The efficiency of the catalyst was compared to the reactivity of a commercial $\mathrm{Cu@charcoal}$ catalyst, which is one of the preferred catalysts to perform click chemistry under thermal conditions. As seen in Table 7, the new material showed great reactivity and high efficiency under both visible light irradiation or dark conditions at $55{ }^{\circ} \mathrm{C}$ (the same temperature as reached upon illumination). Unfortunately, the catalyst showed poor reusability, probably due to the visible leaching of copper species into the solution.

\section{C-C coupling (sp-sp') (Sonogashira)}

One of our recent reports demonstrated that PdNP-decorated materials such as $\mathrm{TiO}_{2}$, nanodiamonds and $\mathrm{Nb}_{2} \mathrm{O}_{5}$ can selectively catalyze the cross-coupling of iodobenzene and

Table 7 Heterogeneous photocatalytic click chemistry catalyzed by CuaNGW ${ }^{a}$

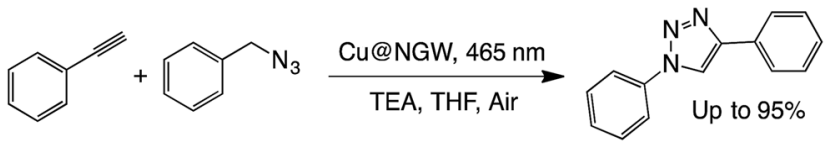

\begin{tabular}{lllll}
\hline & Catalyst & Condition & Time $(\mathrm{h})$ & \% Yield \\
\hline i & Cu@NGW & $465 \mathrm{~nm}, 55^{\circ} \mathrm{C}$ & 6 & $92(95)$ \\
ii & Cu@NGW & Dark, 55 ${ }^{\circ} \mathrm{C}$ & 6 & $93(94)$ \\
iii & Cu@NGW & Dark, RT & 6 & $8(25)$ \\
iv & Cu@NGW & Dark, RT & 24 & 75 \\
iv & Cu@charcoal & Dark, 55 ${ }^{\circ} \mathrm{C}$ & 6 & 21 \\
iv & Cu@charcoal & Dark, 55 ${ }^{\circ} \mathrm{C}$ & 24 & 73 \\
v & NGW & Dark, 55 ${ }^{\circ} \mathrm{C}$ & 6 & ND \\
vi & NGW & Dark, 55 ${ }^{\circ} \mathrm{C}$ & 24 & $2^{d}$
\end{tabular}

${ }^{a}$ Reaction conditions: $15 \mathrm{mg}$ of catalyst, azide/alkyne/TEA (1:1:1), $6 \mathrm{~h}$ under air. ${ }^{b}$ Yields were calculated by ${ }^{1} \mathrm{H}$-NMR analysis in $\mathrm{CDCl}_{3}$ using caffeine as the external standard. ${ }^{c}$ Values between brackets are yields obtained after $6 \mathrm{~h}$ of reaction using supported Cu@NGW*. ${ }^{d}$ Mixture of two isomers. 
Table 8 Light-induced Sonogashira C-C coupling catalyzed by PdaGW ${ }^{a}$

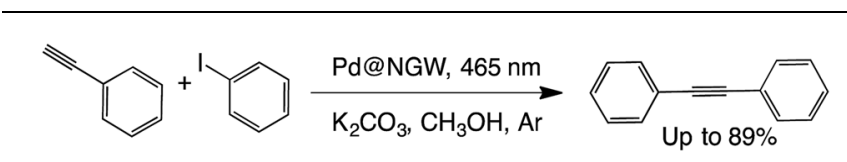

\begin{tabular}{lllll}
\hline & Catalyst & Time (h) & \% Conv & \% Yield \\
\hline i & Pd@NGW* & 5 & $>99(>99)$ & $90(88)$ \\
ii $^{c}$ & Pd@NGW* $_{\text {ii }}$ & 5 & 0 & ND \\
iv & NGW $^{*}$ & 24 & 0 & ND \\
& None & 24 & 0 & ND
\end{tabular}

${ }^{a}$ Reactions conditions: 1 eq. of iodobenzene, 1.3 eq. of phenylacetylene, 2 eq. of $\mathrm{K}_{2} \mathrm{CO}_{3}, 4 \mathrm{~mL}$ methanol, and $60 \mathrm{mg}$ Pd@NGW*. ${ }^{b}$ Yields were determined by GC-FID using $t$-butylbenzene as an external standard. ${ }^{c}$ Under dark conditions, the reaction was run at $42{ }^{\circ} \mathrm{C}$. Values between brackets are yields obtained after $5 \mathrm{~h}$ of reaction using supported Pd@SGW.

phenylacetylene under both reflux and visible light irradiation. ${ }^{32}$ Here we show that the same reaction can be catalyzed by the new Pd@SGW* and Pd@NGW* catalysts and selectively lead to the cross-coupling products showing similar activity. Briefly, the selective cross coupling reaction of iodobenzene and phenylacetylene has been studied with methanol as solvent, $\mathrm{K}_{2} \mathrm{CO}_{3}$ as base and supported PdNPs on the activated and/or functionalized surface of GW (Table 8). Control reactions in the presence of GW and in the absence of catalyst did not show any product after $24 \mathrm{~h}$. The photocatalyst can be reused at least two times with excellent performance (see Table S1 $\dagger$ ), showing the same reactivity as the previously reported materials. ${ }^{32}$

\section{Conclusions}

We show the versatility of the use of glass wool as a very inexpensive, widely available and easily handled material for heterogeneous catalysis. We demonstrate that despite the different nature of the glass wool utilized, the efficacies of at least two commercial materials are similar. We have explored different methods to activate the glass surface in order to better anchor the catalytic species to the material. Also, low surface loadings (ranging from 1 down to $0.07 \mathrm{wt} \%$ ) with different types of metal or metal oxide nanoparticles were tested, to illustrate how easy and reliable these materials are to use. The materials excel in both thermal and light-induced catalysis in a variety of different organic transformations ranging from reductions to $\mathrm{C}-\mathrm{C}$ couplings and cyclizations, showing adaptability to different reaction media and conditions. Also important, the material can be easily separated from the reaction mixture eliminating tedious workup. Notice that optimization of the metal loadings could indeed open the door for more exploratory studies of these materials. This is a first effort on the use of GW as a catalytic support for fine organic reactions and more complex and interesting systems can be developed from here. We envision this as a first step towards an easy way to recover heterogeneous catalysts, and to develop catalysts with great flow chemistry potential.

\section{Experimental}

\section{General}

Unless otherwise specified, all chemicals were purchased from Sigma-Aldrich or Fisher Scientific and used without further purification. Particularly, the following glass wools were used: non-treated GW (Aldrich catalog \#: 20384) and silanized GW (Aldrich catalog \#: 20411). Irgacure-2959 (I-2959) was purchased from Ciba.

Scanning electron microscopy (SEM) images were obtained using a JEOL JSM-1600 SE microscope working at an accelerating voltage of $2 \mathrm{kV}$. X-ray photoelectron spectroscopy (XPS) was performed on a Kratos analytical model Axis Ultra DLD, using monochromatic aluminum K $\alpha$ X-rays at $140 \mathrm{~W}$. XPS data were analyzed using CasaXPS software, Version 2.3.15. All spectra were calibrated at the C 1 s signal at $284.8 \mathrm{eV}$ and fittings obtained using a Gaussian 30\% Laurentian and a Shirley baseline. The amount of metal loaded onto the materials was determined by Inductively Coupled Plasma Optical Emission Spectrometry (ICP-OES), using an Agilent Vista Pro ICP Emission Spectrometer. Approximately $10 \mathrm{mg}$ portions were accurately weighed in triplicate and digested with aqua regia. Solutions were further diluted and measured by ICP-OES. The following emission lines were used for quantification when applicable: Pd 229.65 nm, Au 267.59 nm, Cu $327.00 \mathrm{~nm}$, Co $228.00 \mathrm{~nm}$, and $\mathrm{Ru} 245.55 \mathrm{~nm}$. Diffuse reflectance measurements were carried out using an Agilent Cary 7000 UV-Vis-NIR Universal Measurement Spectrophotometer coupled with an Agilent praying Mantis accessory. UV-Vis spectroscopy was carried out using an Agilent Cary $60 \mathrm{UV}$-Vis Spectrophotometer.

UV irradiation used for catalyst synthesis was performed in a Luzchem photoreactor equipped with UVA bulbs (typically operated with 14 bulbs, corresponding to $\sim 0.029 \mathrm{~W} \mathrm{~cm}^{-2}$ (with $\sim 4 \%$ spectral contamination). Light-emitting diodes (LEDs) of $10 \mathrm{~W}$ from LedEngin were used for photocatalytic reactions in the visible region (centered at 465 and $532 \mathrm{~nm}$, respectively) and in the UV region (centered at $368 \mathrm{~nm}$ ), Fig. $\mathrm{S} 1 . \dagger$

Quantification was carried out using a Perkin Elmer, Claurus Gas Chromatograph coupled to a Flame Ionization Detector (FID) and using a DB-5 column (30 m length, $0.320 \mathrm{~mm}$ diameter, $0.25 \mu \mathrm{m}$ film), helium as a carrier gas and $t$-butyl benzene as the external standard. GC-MS analyses were performed on an Agilent 6890-N Gas Chromatograph with an Agilent 5973 mass selective detector calibrated with acetophenone. All ${ }^{1} \mathrm{H}$ NMR spectra were recorded on a Bruker AVANCE 400 spectrometer expressing the chemical shifts in ppm relative to the $\mathrm{H}$-signal of tetramethylsilane (TMS).

\section{Catalyst preparation}

Glass wool pre-treatments. Two types of glass wool were used for this work: silanized glass wool (SGW) and non-silanized glass wool (NGW). The materials were used as received or treated by different methods described in the ESI. $\dagger$

Metal decoration. Metal or metal oxide nanoparticles were grafted on the activated GW surface using different thermal and photochemical methods. The photochemical method is based 
on a previously described protocol ${ }^{41}$ to synthesize metal NPs utilizing Irgacure 2959 (I-2959) as the photoinitiator. I-2959 can undergo Norrish Type I cleavage upon UVA excitation, generating the corresponding acetyl and ketyl radicals. The latter have been described as strong reducing agents capable of reducing different metals cations $\left(\mathrm{M}^{n+}\right)$ into the resultant metal $\left(\mathrm{M}^{0}\right)$ as shown in Scheme S1. $\dagger$ In general, $500 \mathrm{mg}$ of activated GW (SGW or NGW) and the corresponding amount of metal precursor were mixed together in $200 \mathrm{~mL}$ of Milli-Q water and sonicated. $10 \mathrm{~mL}$ of an ethanolic solution of I-2959 was added and irradiated under UVA for $\sim 1 \mathrm{~h}$. Detailed synthetic methodologies are described in the ESI. $\dagger$

Although some metals are in the form of metal oxides upon deposition on the GW surface, we will refer to all materials as M@GW. In many cases metal oxide nanoparticles are formed spontaneously following ambient exposure of metal nanoparticles.

\section{Catalytic reactions}

Reductive dehalogenation. $60 \mathrm{mg}$ of catalyst (Co@SGW, Pd@SGW or SGW) was dispersed in $5 \mathrm{~mL}$ of methanol in a clean quartz tube, and then $0.2 \mathrm{mmol}$ of methyl 4-halobenzoate and $0.36 \mathrm{mmol}$ of $\mathrm{K}_{2} \mathrm{CO}_{3}$ were added. The reaction mixture was purged with Ar for $10 \mathrm{~min}$ and then irradiated with a $368 \mathrm{~nm}$ LED light source working at $0.4 \mathrm{~W} \mathrm{~cm}^{-2}$. The solid catalyst was separated by filtration. Quantification of the product was done by GC-FID using $t$-stilbene as the external standard. Time of irradiation varies depending on the aryl halides.

\section{Nitrobenzene reductions}

\section{Aniline formation}

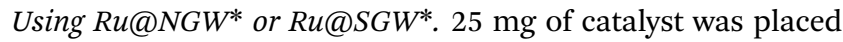
in a centrifuge tube together with $1.5 \mathrm{~mL}$ of nitrobenzene $(1 \mathrm{M})$, $1.7 \mathrm{~mL}$ of EtOH, $0.3 \mathrm{~mL}$ of internal standard 1,3,5-trimethoxy benzene $(0.5 \mathrm{M})$ and $1.5 \mathrm{~mL}$ of $\mathrm{NH}_{2} \mathrm{NH}_{2}$ solution $(6 \mathrm{M})$. The tube was tightly closed and immersed in an oil bath at $78{ }^{\circ} \mathrm{C}$ under constant stirring. Aliquots of $15 \mu \mathrm{L}$ were taken from the reaction mixture, and diluted with $1.5 \mathrm{~mL}$ of ethylacetate prior to GC-FID quantification.

Using $A u @ N G W^{*} .60 \mathrm{mg}$ of Au@NGW* was dispersed in $5 \mathrm{~mL}$ of EtOH in a clean dry round bottom flask then $1 \mathrm{mmol}$ of nitrobenzene and $5 \mathrm{mmol}$ of hydrazine were added. The resulting mixture was heated up to $78{ }^{\circ} \mathrm{C}$ under continuous stirring. The progress of the reaction was monitored by UVvisible spectroscopy. No reaction was detected under these conditions.

Azobenzene formation. $60 \mathrm{mg}$ of Au@NGW* (or Ru@NGW) was dispersed in $5 \mathrm{~mL}$ of i-propanol in a clean glass tube and then $0.03 \mathrm{mmol}$ of $\mathrm{KOH}$ and $0.3 \mathrm{mmol}$ of nitrobenzene were added. The resulting mixture was sonicated for $5 \mathrm{~min}$ prior to irradiation using a $532 \mathrm{~nm}$ LED. The solid catalyst was removed by filtration and quantification was done by UV-visible spectroscopy (Fig. S4 $\uparrow$ ). Notice that no reaction was detected under these conditions when Ru@NGW was tested as the catalyst.

\section{Isomerization/hydrogenation of estragol}

Thermal-induced isomerization. $50 \mathrm{mg}$ of $\mathrm{Ru} @ \mathrm{NGW}^{*}$ (or Ru@SGW*) was dispersed in $8 \mathrm{~mL}$ of i-propanol by sonication ca. for $5 \mathrm{~min}$ in a clean round bottom flask, and then $25 \mu \mathrm{L}(0.16$ $\mathrm{mmol}$ ) of reactant was added. The reaction mixture was heated up to $85{ }^{\circ} \mathrm{C}$ (solvent boiling point) under air with continuous stirring for 24 hours. The progress of the reaction was monitored by GC-FID. The solid catalyst was separated by filtration and the crude product was obtained after solvent evaporation. Quantification was done by GC-FID.

Light-induced isomerization or hydrogenation. $60 \mathrm{mg}$ of Pd@NGW*(or Pd@SGW) was dispersed in $4 \mathrm{~mL}$ of methanol in a clean glass tube, and then $0.14 \mathrm{mmol}$ of estragol was added. The reaction mixture was purged with argon for $15 \mathrm{~min}$ and then irradiated with a $465 \mathrm{~nm}$ LED (a $368 \mathrm{~nm}$ LED for hydrogenation) for 24 hours at room temperature. The progress of the reaction was monitored by GC-FID. The solid catalyst was separated by filtration, and the crude product was obtained after solvent evaporation. Quantification was done either by $1 \mathrm{H}^{-}$ NMR or by GC-FID.

Unfortunately, no reaction was detected under neither of these conditions.

\section{C-C coupling (sp-sp')}

Thermal-induced Sonogashira $C-C$ coupling. $60 \mathrm{mg}$ of Pd@NGW* (Pd@SGW or Ru@NGW) was dispersed in $4 \mathrm{~mL}$ of methanol by sonication for about $5 \mathrm{~min}$ in a clean round bottom flask. Then $0.12 \mathrm{mmol}$ of iodobenzene, $0.16 \mathrm{mmol}$ of phenylacetylene and $0.24 \mathrm{mmol}$ of $\mathrm{K}_{2} \mathrm{CO}_{3}$ were added. The reaction mixture was purged with $\mathrm{Ar}$ and heated up to $42{ }^{\circ} \mathrm{C}$ (temperature of the reaction mixture under blue LED irradiation) with continuous stirring for 5 hours. The solid catalyst was separated by filtration and the crude product was obtained after solvent evaporation. Quantification was done by GC-FID.

Light-induced Sonogashira C-C coupling. $60 \mathrm{mg}$ of Pd@NGW* (Pd@SGW or Ru@NGW) was dispersed in $4 \mathrm{~mL}$ of methanol in a $10 \mathrm{~mL}$ clean glass tube, and then $0.12 \mathrm{mmol}$ of iodobenzene, $0.16 \mathrm{mmol}$ of phenylacetylene and $0.24 \mathrm{mmol}$ of $\mathrm{K}_{2} \mathrm{CO}_{3}$ were added. The reaction mixture was purged with $\mathrm{Ar}$ for $15 \mathrm{~min}$ and then irradiated with a $465 \mathrm{~nm}$ LED light source setup at $1 \mathrm{~W}$ $\mathrm{cm}^{-2}$ for 5 hours. The solid catalyst was separated by filtration and the crude product was obtained after solvent evaporation. Quantification was done by GC-FID. Control experiments were carried out as mentioned above. Notice that no reaction was detected under these conditions when using Ru@NGW as the catalyst; only Pd@NGW* was effective.

$\mathrm{N}-\mathrm{C}$ heterocycloaddition. We followed a previously reported protocol. ${ }^{7}$ Briefly, $15 \mathrm{mg}$ of $\mathrm{Cu} @ \mathrm{NGW}$ (or $\mathrm{Cu} @ \mathrm{NGW}^{*}$ ) was dispersed in $1.5 \mathrm{~mL}$ of THF in a quartz test tube and mixed with azide $(0.5 \mathrm{mmol})$, alkyne $(0.5 \mathrm{mmol})$, and amine $(0.5 \mathrm{mmol})$. The reaction mixture was stirred and irradiated with a $465 \mathrm{~nm}$ LED at room temperature and under air for $6 \mathrm{~h}$. The solid catalyst was separated by filtration and the pure product was obtained after vacuum evaporation. Yields were calculated by ${ }^{1} \mathrm{H}$ NMR in $\mathrm{CDCl}_{3}$.

Control experiments were performed under dark conditions at room temperature or at $55{ }^{\circ} \mathrm{C}$ (maximum temperature reached upon illumination) under air.

$C-C$ coupling $\left(s p^{3}-s p^{3}\right)$ : dimerization of benzyl bromides. We followed a previously reported protocol. ${ }^{7}$ Briefly, $30 \mathrm{mg}$ of $\mathrm{Au} @ \mathrm{NGW}^{*}$ was dispersed in $4 \mathrm{~mL}$ of dichloromethane (DCM) 
in a glass test tube and then $0.1 \mathrm{mmol}$ of 4-nitrobenzylbromide and $0.2 \mathrm{mmol}$ of diisopropyl ethylamine (DIPEA) were added. The resulting mixture was purged with argon for 15 minutes prior to $532 \mathrm{~nm}$ LED irradiation with a 4xLED system each working at $0.56 \mathrm{~W} \mathrm{~cm}^{-2}$. The solid catalyst was separated by filtration and the product was obtained after vacuum evaporation. Yields and conversions were calculated by ${ }^{1} \mathrm{H}$ NMR in $\mathrm{CDCl}_{3}$ using caffeine as the external standard. Control experiments were carried out as mentioned above.

\section{Catalyst recyclability}

Co@SGW, Pd@NGW* and Au@NGW* catalysts were tested for potential reusability in the direct, selective and efficient reductive dehalogenation, Sonogashira coupling and 4-nitrobenzyl bromide dimerization respectively, using the conditions described above within at least two reusability cycles. The catalyst was removed from the reaction mixture by filtration, washed twice with methanol and 3 times with water, and then dried in an oven at $100{ }^{\circ} \mathrm{C}$ overnight prior to reuse (Table $\mathrm{S} 1 \dagger$ ).

\section{Conflicts of interest}

There are no conflicts to declare.

\section{Acknowledgements}

This work was supported by the Natural Sciences and Engineering Research Council of Canada, the Canada Foundation for Innovation, the Canada Research Chairs Program and funding from Canada's International Development Research Centre (IDRC). Thanks are due to the RISE program for the scholarship awarded to M. C. and the Generalitat Valenciana (BEST/2017/049) for the financial support granted to M. L. M. The authors would like to thank Dr Yun Liu for helping on the acquisition of the SEM images.

\section{References}

1 I. W. Davies, L. Matty, D. L. Hughes and P. J. Reider, J. Am. Chem. Soc., 2001, 123, 10139-10140.

2 R. Schlçgl, Angew. Chem., Int. Ed., 2015, 54, 3465-3520.

3 W. C. Conner and J. L. Falconer, Chem. Rev., 1995, 95, 759788.

4 A. Elhage, A. E. Lanterna and J. C. Scaiano, ACS Catal., 2017, 7, 250-255.

5 D. Cambie, C. Bottecchia, N. J. W. Straathof, V. Hessel and T. Noel, Chem. Rev., 2016, 116, 10276-10341.

6 A. E. Lanterna, A. Elhage and J. C. Scaiano, Catal. Sci. Technol., 2015, 5, 4336-4340.

7 B. Wang, J. Durantini, J. Nie, A. E. Lanterna and J. C. Scaiano, J. Am. Chem. Soc., 2016, 138, 13127-13130.

8 Sigma-Aldrich, Glass Wool, http://www.sigmaaldrich.com/ catalog/product/supelco/20411?lang $=$ en\&region $=$ CA, accessed September, 2017, 2017.

9 B. Steyn, M. C. Oosthuizen, R. MacDonald, J. Theron and V. S. Brozel, Proteomics, 2001, 1, 871-879.
10 M. Nisnevitch, M. Kolog-Gulco, D. Trombka, B. S. Green and M. A. Firer, J. Chromatogr. B: Biomed. Sci. Appl., 2000, 738, 217-223.

11 Y. Matatov-Meytal and M. Sheintuch, Appl. Catal., A, 2002, 231, 1-16.

12 V. V. Barelko, M. V. Kuznetsov, V. G. Dorokhov and I. Parkin, Russ. J. Phys. Chem. B, 2017, 11, 606-617.

13 R. W. Macdonald and K. E. Hayes, J. Chem. Soc., Chem. Commun., 1972, 1030.

14 G. K. Ramaswamy, A. Somasundaram, B. K. Kuppuswamy and M. Velayudham, J. Chin. Chem. Soc., 2013, 60, 97-102.

15 H. T. Yang, Z. P. Fang, X. Y. Fu and L. F. Tong, Chin. J. Catal., 2007, 28, 947-952.

16 B. S. Bal'zhinimaev, A. P. Suknev, Y. K. Gulyaeva and E. V. Kovalyov, Catalysis in Industry, 2015, 7, 267-274.

17 L. G. Simonova, V. V. Barelko, A. V. Toktarev, A. F. Chernyshov, V. A. Chumachenko and B. S. Bal'zhinimaev, Kinet. Catal., 2002, 43, 61-66.

18 A. I. Carrillo, K. G. Stamplecoskie, M. L. Marin and J. C. Scaiano, Catal. Sci. Technol., 2014, 4, 1989-1996.

19 B. H. R. Suryanto, X. Y. Lu, H. M. Chan and C. Zhao, RSC Adv., 2013, 3, 20936-20942.

20 D. J. Morgan, Surf. Interface Anal., 2015, 47, 1072-1079.

21 K. C. Park, I. Y. Jang, W. Wongwiriyapan, S. Morimoto, Y. J. Kim, Y. C. Jung, T. Toya and M. Endo, J. Mater. Chem., 2010, 20, 5345.

22 C. Bock, C. Paquet, M. Couillard, G. A. Botton and B. R. MacDougall, J. Am. Chem. Soc., 2004, 126, 8028-8037.

23 J. P. Espinos, J. Morales, A. Barranco, A. Caballero, J. P. Holgado and A. R. Gonzalez-Elipe, J. Phys. Chem. B, 2002, 106, 6921-6929.

24 A. Y. Klyushin, T. C. R. Rocha, M. Havecker, A. Knop-Gericke and R. Schlogl, Phys. Chem. Chem. Phys., 2014, 16, 78817886.

25 C. S. Higman, A. E. Lanterna, M. L. Marin, J. C. Scaiano and D. E. Fogg, ChemCatChem, 2016, 8, 2446-2449.

26 R. B. Bedford, C. S. J. Cazin and D. Holder, Coord. Chem. Rev., 2004, 248, 2283-2321.

27 CRC Handbook of Chemistry and Physics, ed. D. R. Lide, Taylor and Francis Group, Boca Raton, FL, 88th edn, 2007, p. 2640.

28 B. Sahoo, A. E. Surkus, M. M. Pohl, J. Radnik, M. Schneider, S. Bachmann, M. Scalone, K. Junge and M. Belle, Angew. Chem., 2017, 129, 11394-11399.

29 J. J. Devery, J. D. Nguyen, C. Dai and C. R. J. Stephenson, ACS Catal., 2016, 6, 5962-5967.

30 L. B. Liao, Q. H. Zhang, Z. H. Su, Z. Z. Zhao, Y. N. Wang, Y. Li, X. X. Lu, D. G. Wei, G. Y. Feng, Q. K. Yu, X. J. Cai, J. M. Zhao, Z. F. Ren, H. Fang, F. Robles-Hernandez, S. Baldelli and J. M. Bao, Nat. Nanotechnol., 2014, 9, 69-73.

31 A. S. Hainer, J. S. Hodgins, V. Sandre, M. Vallieres, A. E. Lanterna and J. C. Scaiano, ACS Energy Lett., 2018, 3, 542-545.

32 A. Elhage, A. E. Lanterna and J. C. Scaiano, ACS Sustainable Chem. Eng., 2018, 6, 1717-1722.

33 A. Elhage, A. E. Lanterna and J. C. Scaiano, ACS Catal., 2017, 7, 250-255. 
34 P. Roy, A. P. Periasamy, C. T. Liang and H. T. Chang, Environ. Sci. Technol., 2013, 47, 6688-6695.

35 B. W. Zhou, J. L. Song, H. C. Zhou, L. Q. Wu, T. B. Wu, Z. M. Liu and B. X. Han, RSC Adv., 2015, 5, 36347-36352.

36 H. Y. Zhu, X. B. Ke, X. Z. Yang, S. Sarina and H. W. Liu, Angew. Chem., Int. Ed., 2010, 49, 9657-9661.

37 K. Selvam, H. Sakamoto, Y. Shiraishi and T. Hirai, New J. Chem., 2015, 39, 2856-2860.
38 S. Sarina, E. R. Waclawik and H. Y. Zhu, Green Chem., 2013, 15, 1814-1833.

39 L. B. Wang, X. Q. Pan, Y. Zhao, Y. Chen, W. Zhang, Y. F. Tu, Z. B. Zhang, J. Zhu, N. C. Zhou and X. L. Zhu, Macromolecules, 2015, 48, 1289-1295.

40 H. M. D. Bandara and S. C. Burdette, Chem. Soc. Rev., 2012, 41, 1809-1825.

41 K. L. McGilvray, M. R. Decan, D. Wang and J. C. Scaiano, J. Am. Chem. Soc., 2006, 128, 15980-15981. 